\title{
MST radar signal processing using iterative adaptive approach
}

\author{
C. Raju ${ }^{* \dagger}$ and T. Sreenivasulu Reddy ${ }^{\dagger}$
}

\begin{abstract}
Power spectrum is the considerable aspect in the atmospheric radar data processing to estimate wind parameters. Due to the poor resolution and high sidelobe level problems of the existing algorithms, there is a requisite for the novel data-dependent approaches. A non-parametric and hyperparameter-free iterative adaptive approach (IAA) is presented for the power spectral density estimation. This approach is able to work with single snapshot and is obtained by minimizing the weighted least square fitting criterion. The IAA method provides the accurate amplitude and frequency estimation for the simulated data. The data for the above study is collected from Indian MST (mesosphere, stratosphere, and troposphere) radar. The power spectrum and Doppler frequency are estimated using IAA. In this paper, zonal $(U)$, meridional $(V$, windspeed $(W)$ are also calculated and validated using Global Positioning System Sonde data. The effectiveness of the spectral estimation performance showed by IAA is demonstrated and assessed.
\end{abstract}

Keywords: Spectral estimation, MST radar, IAA, GPS

\section{Background}

Indian MST radar provides information on wind data above $3.5 \mathrm{~km}$ with a resolution of $150 \mathrm{~m}$. The three wind components $U, V$ and $W$ are determined by the Doppler beam swinging (DBS) method of the MST radar. The radar collects the data using multiple beam positions with $16 \mu$ s coded pulse and with an inter pulse period (IPP) of $100 \mu \mathrm{s}$. The online calculation of Doppler power spectra for each range bin can be obtained by subjecting the complex time series data to the process of fast Fourier transform (FFT). The DC removal, average noise level estimation, interference removal and incoherent integration are the steps that are involved in offline data processing. The 0th, 1st and 2nd moments denotes the signal strength, mean Doppler shift and half width parameters of the spectrum are computed, respectively.

The accurate estimation of the Doppler frequency is the crucial one in the detection and estimation of the wind speed by the MST radar. A package for processing the radar data has been developed by the National

\footnotetext{
*Correspondence: c.raju1131@gmail.com

${ }^{\dagger} \mathrm{C}$. Raju and T. Sreenivasulu Reddy have equally contributed for the paper Department of ECE, Sri Venkateswara University College of Engineering, S.V. University, Tirupati, A.P., India
}

Atmospheric Research Laboratory (NARL), Gadanki, Andhra Pradesh, India. It is known as the atmospheric data processor (ADP) (Anandan 2002). The Doppler frequencies can be accurately estimated by the ADP up to certain heights. Since the signals are highly corrupted with noise at higher altitudes, the ADP is unable to estimate the Doppler frequencies and thus the wind speed. It can be seen in the literature that several algorithms have been put to use to accurately estimate the Doppler frequencies from MST radar data.

Bispectral estimation algorithm (Anandan et al. 2001) is applied to radar at a high computational cost. The multitaper-based spectral estimation (Anandan et al. 2004) has the advantage of reduction in variance and has been applied to the radar data. However, it has spectral peak broadening. An adaptive estimation technique has been presented to estimate the Doppler spectra, with certain parameters to adaptively track the signal in the range of Doppler spectral frame (Anandan et al. 2005). Several methods like wavelet-based denoising (Thatiparthi et al. 2009) and cepstral thresholding (Reddy and Reddy 2010a, b) have also been used for spectrum cleaning and then Doppler spectrum estimation leading to the calculation of wind velocities. According to (Reddy and Reddy 2010a, b), a polyphase approach was employed for 
spectrum estimation using uniform filter banks. Recent studies applied principal component analysis (PCA) on the radar data, prior to periodogram estimation using Blackman-Tukey, and minimum variance methods (Uma Maheswara Rao et al. 2014). All the existing methods used in spectral estimation for atmospheric radar data falls under two estimation methods either parametric or nonparametric. However, parametric method requires the prior knowledge of some parameters and nonparametric method has global leakage (peaks at unwanted frequencies) and local leakage (main beam limits). In the present study, the iterative adaptive approach (IAA) (Yardibi et al. 2010) is put into use to estimate the spectrum of MST radar data collected from NARL. The IAA algorithm is based on weighted least squares minimization and is found to give excellent results for simulated data and real-time data.

In this paper, lowercase and uppercase boldface letters represent vectors and matrices, respectively. Normal letters are used to indicate scalar quantities. Notations $|\cdot|$, $\|\cdot\|,(\cdot)^{*},(\cdot)^{T}$, and $E(\cdot)$ denote the modulus, the Frobenius norm, the complex conjugate transpose (Hermitian transpose), the transpose and the expectation operator. Subscript $[\cdot]_{k}$ denotes the $k$ th element of a vector, and $\boldsymbol{I}_{N}$ represents an identity matrix of size $N$.

\section{Data model}

The MST radar data collected from the NARL is a uniformly spaced complex baseband signal consisting of inphase $(I)$ and quadrature $(Q)$ phase components.

Let $\left\{y_{n}\right\}_{n=1}^{N}$ be the complex data obtained by weighted combination of $C$ complex exponentials with frequencies $\left\{\Omega_{r}\right\}_{r=1}^{N} \in\left[0, \Omega_{\max }\right]$

$$
y_{n}=\sum_{r=1}^{C} q_{r} e^{j \omega_{r} t_{n}}+e_{n}
$$

where $C$ is a small number, $\left\{t_{n}\right\}_{n=1}^{N}$ denotes the sampling time instants which can be non-uniformly spaced. $q_{r}$ is the magnitude associated with $r$-th frequency component $\Omega_{r}, e_{n}$ is the additive white Gaussian noise component corresponding to the $n$-th sampling time.

Consequently, the complex data signal can be modelled as

$$
y_{n}=\sum_{r=1}^{R} q_{r} e^{j \omega_{r} t_{n}}+e_{n}
$$

The expanded version of the above equation is

$$
\left[\begin{array}{c}
y_{1} \\
\vdots \\
y_{N}
\end{array}\right]=\left[\begin{array}{ccc}
e^{j \omega_{1} t_{1}} & \cdots & e^{j \omega_{R} t_{1}} \\
\vdots & & \vdots \\
e^{j \omega_{1} t_{N}} & \cdots & e^{j \omega_{R} t_{N}}
\end{array}\right]\left[\begin{array}{c}
q_{1} \\
\vdots \\
q_{R}
\end{array}\right]+\left[\begin{array}{c}
e_{1} \\
\vdots \\
e_{N}
\end{array}\right]
$$

Those values of $r$ for which $\omega_{r} \in\left\{\Omega_{r}\right\}_{r=1}^{N}$, the corresponding $q_{r}$ values will be non-zero. The equation can vectorially be represented in the following form:

$$
y=D q+e
$$

where $\boldsymbol{y}=\left[y_{1}, y_{2}, \ldots, y_{N}\right]^{T}, \boldsymbol{D}=\left[\boldsymbol{d}\left(\omega_{1}\right), \boldsymbol{d}\left(\omega_{2}\right), \ldots, \boldsymbol{d}\left(\omega_{N}\right)\right]^{T}$ with

$$
\boldsymbol{d}\left(\omega_{r}\right)=\left[e^{j \omega_{1} t_{1}}, \ldots, e^{j \omega_{r} t_{N}}\right]=\boldsymbol{d}_{\boldsymbol{r}}
$$

$\boldsymbol{q}=\left[q_{1}, q_{2}, \ldots, q_{R}\right]^{T},\left|q_{r}\right|^{2}$ is the power value associated with the $r$-th frequency component that has to be estimated.

\section{Iterative adaptive approach}

Iterative adaptive approach is a weighted least squarebased data-dependent, non parametric algorithm. It can be used for the single data sequence or the multiple data snapshots spectral estimation. Here, we assume the single snapshot case.

The covariance matrix of the received signal is given by the following model

$$
\boldsymbol{R}=E\left(\boldsymbol{y} y^{*}\right)=\sum_{r=1}^{R}\left|q_{r}\right|^{2} \boldsymbol{d}_{r} \boldsymbol{d}_{r}^{*}+E\left(\boldsymbol{e}^{*}\right)=\boldsymbol{D P} \boldsymbol{D}^{*}
$$

where

$$
\begin{aligned}
& E\left(\boldsymbol{e} \boldsymbol{e}^{*}\right)=\operatorname{diag}\left(\sigma_{1}, \sigma_{2}, \ldots, \sigma_{N}\right) \\
& \boldsymbol{P}=\operatorname{diag}\left(\left|q_{1}\right|^{2},\left|q_{2}\right|^{2}, \ldots,\left|q_{R}\right|^{2}, \sigma_{1}, \sigma_{2}, \ldots, \sigma_{N}\right) \\
& \quad \triangleq \operatorname{diag}\left(m_{1}, m_{2}, \ldots, m_{R+N}\right)
\end{aligned}
$$

where $\left\{m_{i}\right\}_{i=1}^{R+N}$ is a replacement for $\left\{\left|q_{i}\right|^{2}\right\}_{i=1}^{R+N}$. The first $R$ diagonal elements of the matrix $\boldsymbol{P}$ denote the power values that are to be estimated and the remaining $N$ diagonal elements denote the noise variance values.

The IAA algorithm is based on the minimization of weighted least squares fitting criterion,

$$
f=\left\|\boldsymbol{y}-\boldsymbol{d}_{r} m_{r}\right\|_{\boldsymbol{O}_{r}^{-1}}^{2}, \quad r=1,2, \ldots, R+N
$$

where $\|\boldsymbol{X}\|_{\boldsymbol{O}_{r}^{-1}}^{2} \triangleq \boldsymbol{X}^{H} \boldsymbol{O}_{r}^{-1} \boldsymbol{X} . \boldsymbol{O}_{\boldsymbol{r}}$ is the interference covariance matrix written as

$$
\boldsymbol{O}_{\boldsymbol{r}}=E\left\{\left(\boldsymbol{y}-\boldsymbol{d}_{r} \mathrm{~m}_{r}\right)\left(\boldsymbol{y}-\boldsymbol{d}_{r} \mathrm{~m}_{r}\right)^{H}\right\}=\boldsymbol{R}-\mathrm{m}_{r} \boldsymbol{d}_{r} \boldsymbol{d}_{r}^{*}
$$

Let $m_{r}(i)$ denotes the estimate of $m_{r}$ at $i$ th iteration, and let $\boldsymbol{R}(i)$ be the covariance matrix $\boldsymbol{R}$ derived from $\left\{m_{i}\right\}_{i=1}^{R+N}$.

Minimizing the Eq. (9) with respect to $\mathrm{m}_{r}, r=1,2, \ldots, R+N$, yields 


$$
\hat{m}_{r}(i+1)=\frac{\boldsymbol{d}_{r}^{*} \boldsymbol{O}_{r}^{-1} \boldsymbol{y}}{\boldsymbol{d}_{r}^{*} \boldsymbol{O}_{r}^{-1} \boldsymbol{d}_{r}}, \quad r=1,2, \ldots, R+N
$$

Making use of the Woodbury matrix identity of

$$
\boldsymbol{d}_{r}^{*} \boldsymbol{O}_{r}^{-1}=\frac{\boldsymbol{d}_{r}^{*} \boldsymbol{R}^{-1}(i)}{1-\left|m_{r}(i)\right| \boldsymbol{d}_{r}^{*} \boldsymbol{R}^{-1}(i) \boldsymbol{d}_{r}}
$$

The estimation of the power by means of the following iterative process

$$
\hat{m}_{r}(i+1)=\frac{\boldsymbol{d}_{r}^{*} \boldsymbol{R}^{-1}(i) \boldsymbol{y}}{\boldsymbol{d}_{r}^{*} \boldsymbol{R}^{-1}(i) \boldsymbol{d}_{r}}, \quad r=1,2, \ldots, R+N
$$

The initial estimate $m_{r}(0)$ can be obtained using the single frequency least-squares (SFLS) method (which is known as periodogram).

$$
m_{r}(0)=\frac{\left|\boldsymbol{d}_{r}^{*} \boldsymbol{y}\right|^{2}}{\left\|\boldsymbol{d}_{r}\right\|^{4}}, \quad r=1,2, \ldots, R+N .
$$

The algorithm is summarized in Table 1.

\section{Results}

\section{Simulation}

The simulation results for the complex data explained in Data Model are displayed. With $N=200$ and $C=3$, the data samples are generated from a signal consisting of three exponentials at $0.3100,0.3150,0.1450 \mathrm{~Hz}$ frequencies having the amplitudes $q_{1}=10 e^{j \varphi_{1}}, q_{2}=10 e^{j \varphi_{2}}$, and $q_{3}=10 e^{j \varphi_{3}}$ with a sampling interval of $1 \mathrm{~s}$. The phase values $\left\{\varphi_{r}\right\}_{r=1}^{3}$ are distributed in uniform and independent manner $[0,2 \pi]$. The noise component is introduced in the $\varepsilon$ term, which is the normal white noise with zero mean and $\sigma$ variance. The signal-to-noise ratio (SNR) in $\mathrm{dB}$ for the data model is defined as $\mathrm{SNR}=10 \log \left(\frac{100}{\sigma}\right)$.

The power spectrum of the original test signal generated using the data model before adding noise is depicted in Fig. 1. The spectrum of the signal for $S N R=0 \mathrm{~dB}$ using periodogram and IAA are shown in Fig. 2. The periodogram is obtained by padding the 200 element timeseries data with 312 zeros and performing the 512-point FFT. For IAA the number of processing points $R$ is taken as 512 .

\section{Table 1 Summary of the IAA algorithm}

\begin{tabular}{ll}
\hline S. no. & Operation \\
\hline 1. & Initialize the value $m_{r}(0)$ using the periodogram \\
2. & Compute the covariance matrix $\boldsymbol{R}=\sum_{r=1}^{R+N} m_{r} \boldsymbol{d}_{r} \boldsymbol{d}_{r}^{*}$ \\
3. & Update $\hat{m}_{r}(i+1)$ using the equation for $r=1,2, \ldots, R+N$ \\
4. & Repeat steps $2-3$ until the convergence condition is reached \\
\hline
\end{tabular}

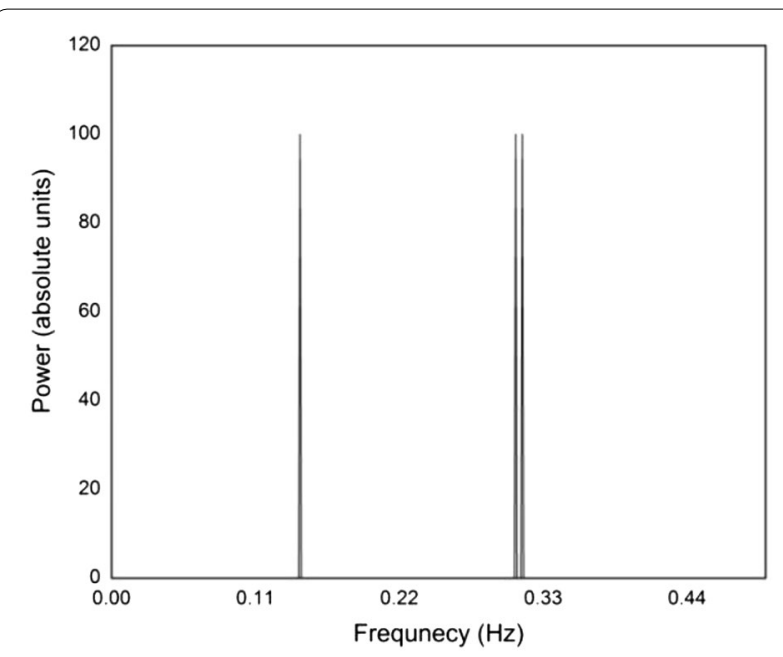

Fig. 1 Spectrum of the original signal
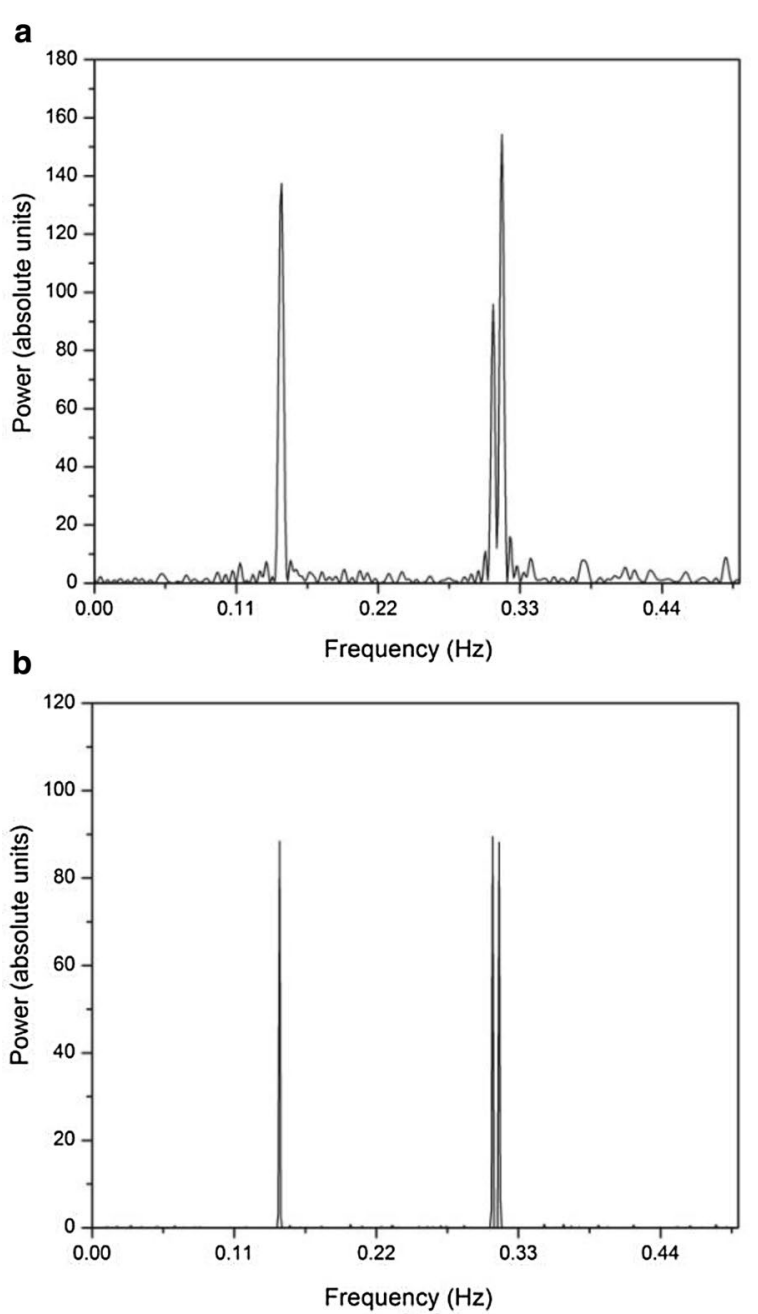

Fig. 2 Spectrum of the test signal for $S N R=0 d B$ a periodogram $\mathbf{b}$ IAA 

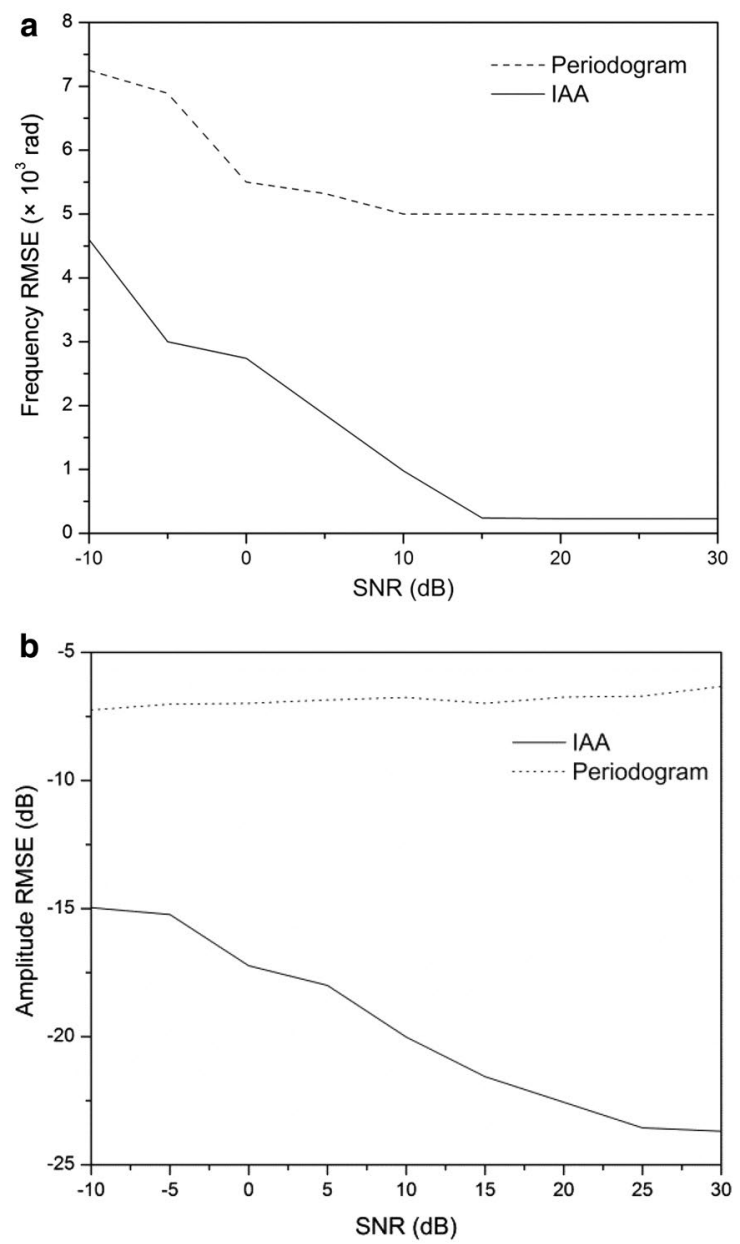

Fig. 3 a RMSE of normalized frequency versus SNR. b Amplitude RMSE versus SNR plot

The root mean square error (RMSE) of the frequency estimation and amplitude estimation versus SNR plots are shown in Fig. 3a, b, respectively. All the performance curves are obtained via 100 Monte Carlo simulations. We change the value of $\sigma^{2}$ to obtain different signal-to-noise ratio conditions. From Fig. 3a, b, it can be observed that both the RMSE of the frequency and the amplitude estimates decreased with the SNR as expected. It can also be observed that the IAA method has better variance characteristics than the Periodogram method. It is evident from the above simulations, that even the signal is completely buried in noise the IAA method is able to retrieve the parameters well.

\section{MST radar data}

The radar data collected from the Indian MST radar being operated at the NARL, Gadanki, Andhra Pradesh is taken for the present study. The MST radar data is one of the e formats of 15 scans with each scan having signal information from six beam directions (East, West, Zenith-X, Zenith-Y, North and South). Each beam consists of 147 height range bins with a resolution of $150 \mathrm{~m}$, starting from $3.6 \mathrm{~km}$ and reaching to a height of $25.6 \mathrm{~km}$. Each range bin contains complex time-series data with 512 samples. The spectrum of the radar signal is calculated using IAA. Since, the echoes are usually corrupted by interference, clutter, etc., it has to be cleaned before analysis. Maximum peak detection method (Anandan et al. 1996) is used for the estimation of Doppler profile after performing spectrum cleaning of the radar signal. The Doppler frequencies are calculated from the Doppler profiles.

Once the Doppler frequencies are computed, the Doppler velocities are found out by multiplying each of the frequencies with $c / 2 f_{c}$ where $c$ is the velocity of light and $f_{c}$ is the operating frequency of the Doppler radar. Both Doppler frequencies and the velocities are calculated for all 6 beams and 147 range bins. Using the Doppler velocities for the 6 beams denoted as $v_{E}, v_{W}, v_{Z X}, v_{Z Y}, v_{N}, v_{S}$ where the subscripts represents the corresponding beams, the three wind velocities components are evaluated as follows

$$
\left[\begin{array}{c}
v_{x} \\
v_{y} \\
v_{z}
\end{array}\right]=\left[\begin{array}{ccc}
0.603 & 0 & 0 \\
0 & 0.603 & 0 \\
0 & 0 & 0.603
\end{array}\right]^{-1} *\left[\begin{array}{c}
0.1736\left(v_{E}-v_{W}\right) \\
0.1736\left(v_{N}-v_{S}\right) \\
0.1736\left(v_{Z X}-v_{Z Y}\right)
\end{array}\right]
$$

where $v_{x}, v_{y}, v_{z}$ are the zonal $U$, meridional $V$, and the vertical $Z$ velocity components. The Zenith-X and Zenith-Y beams are in the vertical direction and do not play a role in the determination of the wind velocity.The Wind speed $W$ is computed as

$$
W=\left(v_{x}^{2}+v_{y}^{2}\right)^{1 / 2}
$$




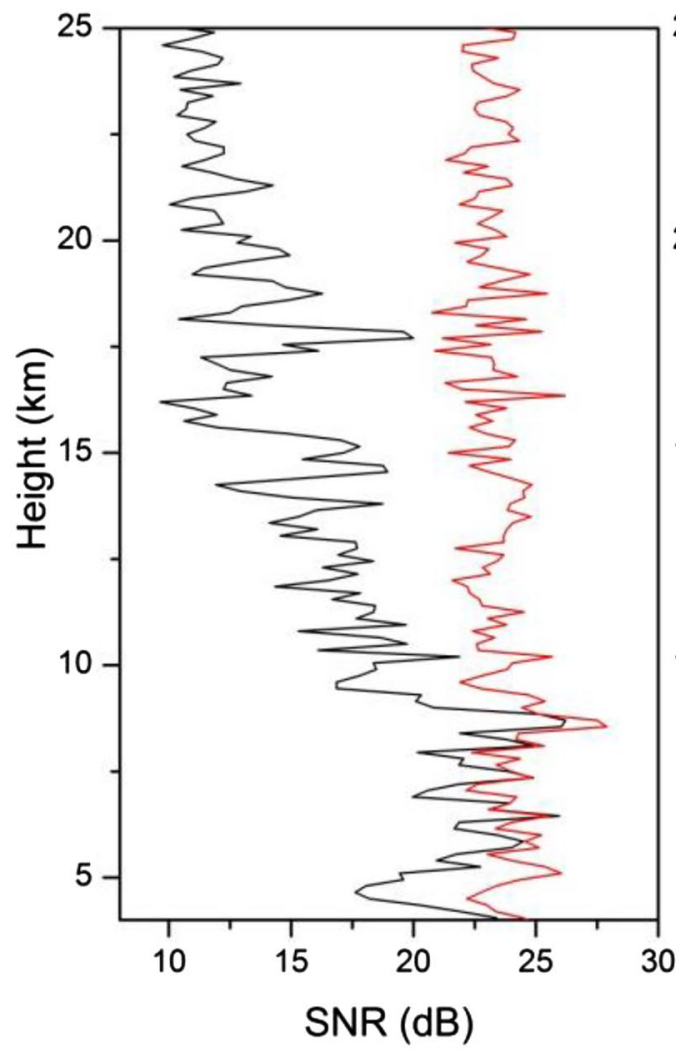

a

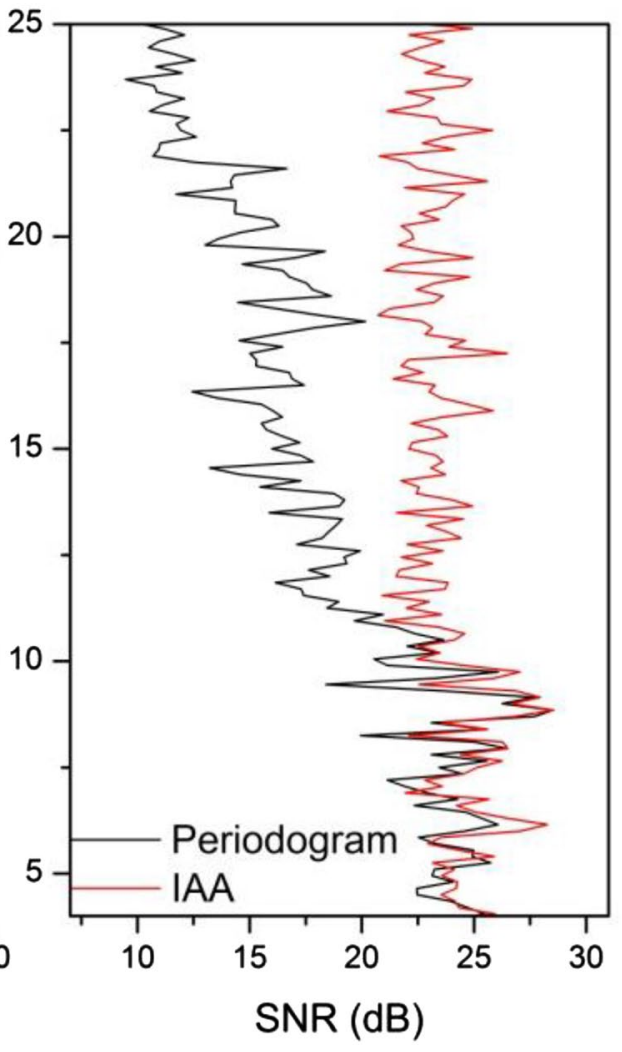

b

Fig. 4 Height profiles of SNR estimated using periodogram and IAA. a East beam; b south beam for data collected on February 9, 2015

The wind speed thus obtained is then compared with the corresponding wind speed collected from the global positioning system (GPS) radiosonde (Jagannadha Rao et al. 2003).

The power spectrum of the collected data is determined using IAA method. The basic method of periodogram is employed for estimating the power spectrum when complex time-series data is subjected to the ADP.
Figure $4 \mathrm{a}, \mathrm{b}$ shows the improvement of output SNR estimated from power spectrum using periodogram and IAA for the east and south beams, respectively, for the MST radar data collected on February 9, 2015. The output SNR is obtained using the method (Hildebrand and Sekhon 1974). The comparison of average SNR values in dB for six beams on February 9 and 10, 2015 for the periodogram and IAA algorithms is given in Table 2. From

Table 2 Comparison of average SNR for periodogram and IAA algorithm

\begin{tabular}{llllllll}
\hline Date & Algorithm & East & West & North & South & Zenith-X & Zenith-Y \\
\hline Feb 9, 2015 & Periodogram & 19.47 & 18.23 & 20.14 & 21.57 & 18.45 & 17.98 \\
& IAA & 23.84 & 22.48 & 22.65 & 24.09 & 22.84 & 21.45 \\
Feb 10, 2015 & Periodogram & 22.35 & 23.41 & 18.56 & 17.86 & 20.85 & 19.81 \\
& IAA & 25.76 & 24.38 & 20.86 & 19.47 & 23.98 & 22.57 \\
\hline
\end{tabular}



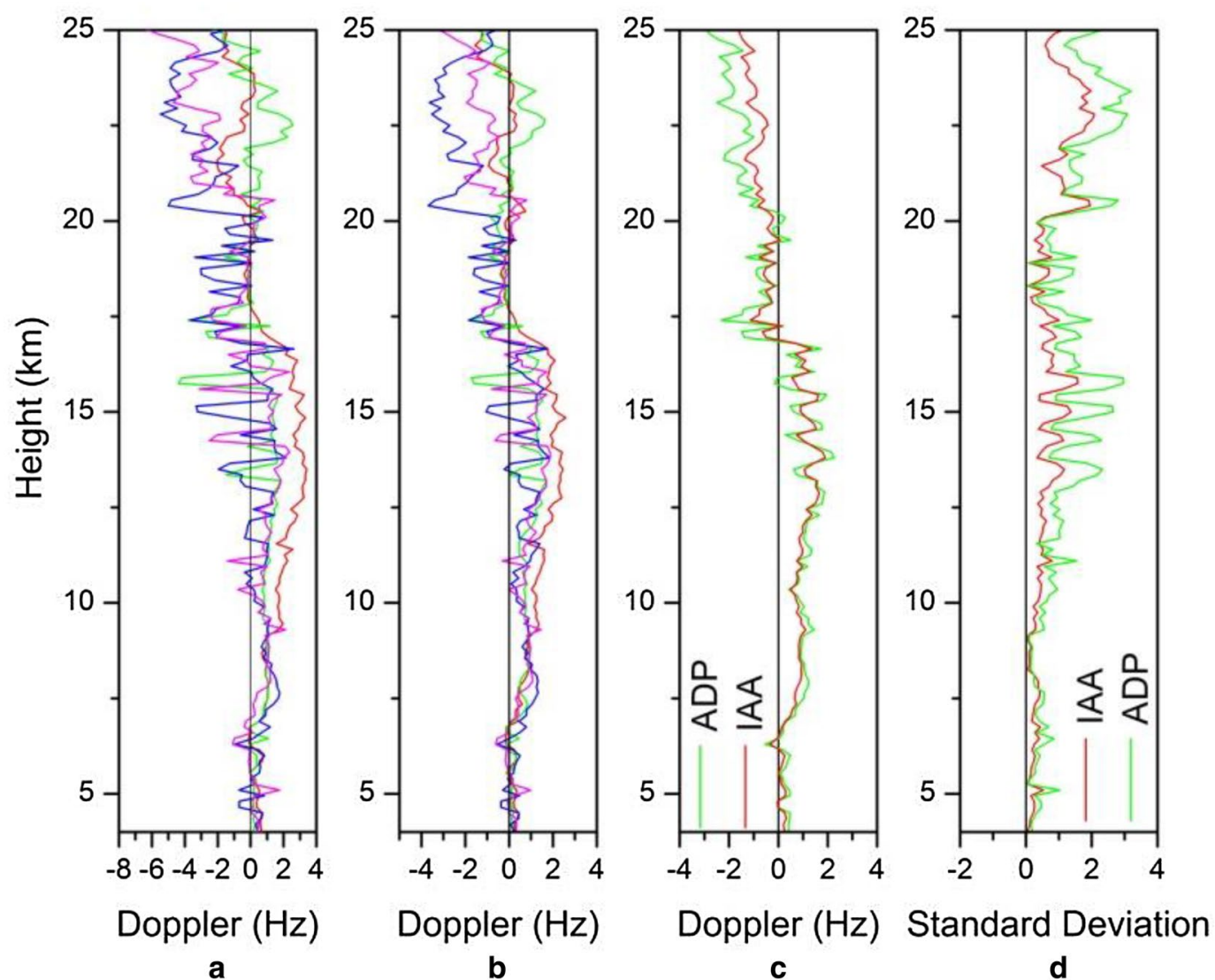

Fig. 5 Doppler height profiles for four scans of the east beam using a ADP, b IAA. c Mean Doppler height profile of the east beam. $\mathbf{d}$ Standard deviation for the east beam for February 9, 2015 data

Table 2, it is seen that the IAA gives the better improvement in SNR values for all the six beams.

The Doppler height profiles for four scans of the east beam attained using ADP and IAA are shown in Fig. 5a, b, respectively, for the radar data collected on February 9, 2015. The compared mean Doppler profiles and standard deviations are shown in Fig. $5 c$, d respectively. The observed significant difference between ADP and IAA is that the standard deviation for IAA lied very close to zero.
The Zonal, Meridional and Wind Speed components calculated using the ADP, IAA and GPS radiosonde are depicted in Figs. 6 and 7 for the data collected on June 9, 2006 and February 9, 2015, respectively. It is revealed that the IAA is following the GPS. The minor deviation from 15 to $17.5 \mathrm{~km}$ can be attributed to the fact that the data used in IAA are collected from the reflected echoes from the layers of the atmosphere in the vertical direction without any drift in the horizontal direction, whereas in the data collected through GPS, there can be horizontal 

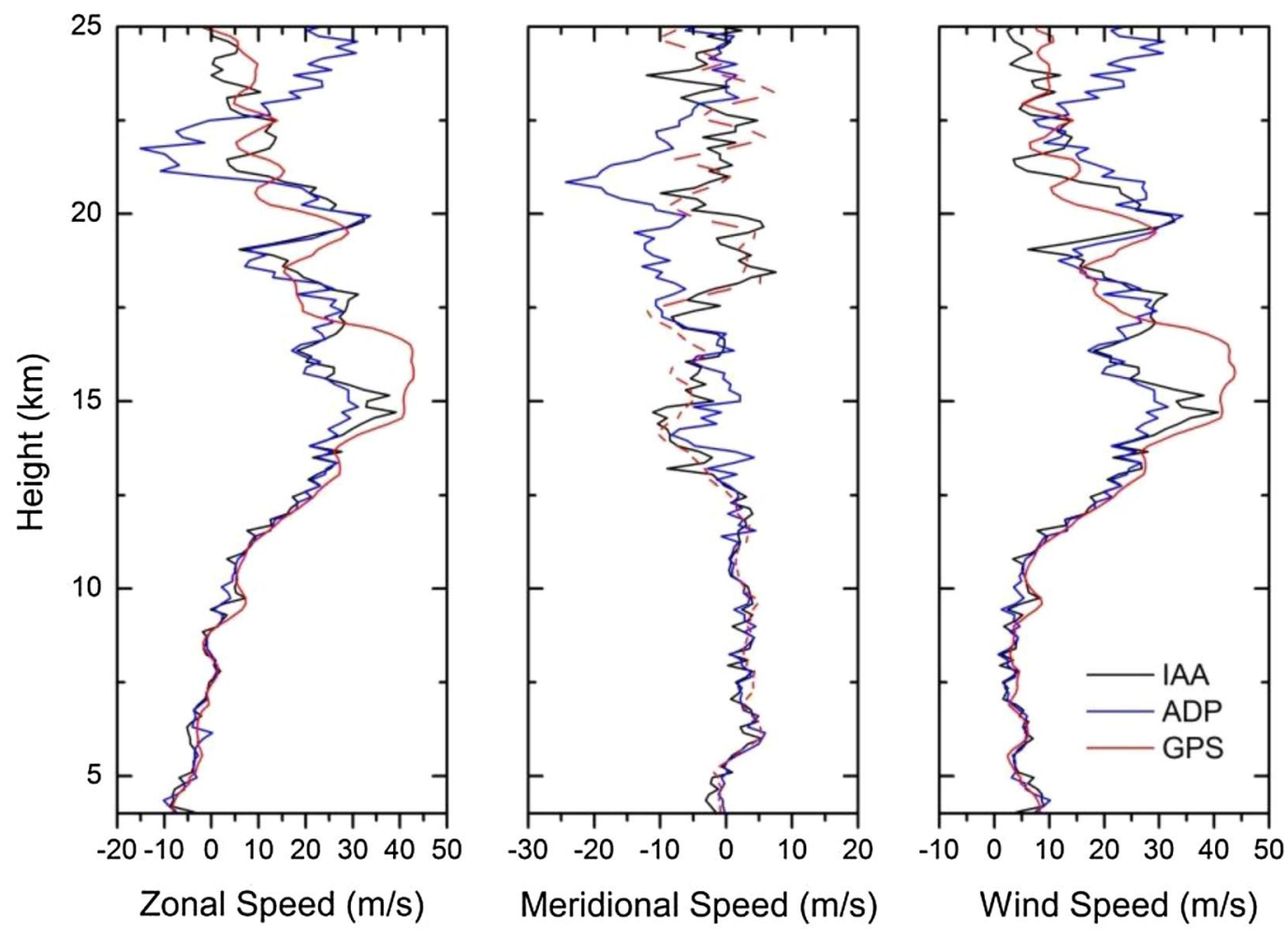

Fig. 6 Zonal, Meridional and Wind Speed for June 9, 2006 data using ADP, IAA and GPS radiosonde

drift of the balloon due to high wind speeds. The wind speeds using ADP, IAA and GPS radiosonde for real-time radar data collected on two different dates namely July 2, 2014 and February 9, 2015 is represented in Fig. 8.

The consistency of the algorithm is checked by calculating the correlation between GPS radiosonde data and IAA wind speeds for the radar data collected during 4th-8th October, 2006 and 9th-12th February, 2015. A significant correlation coefficient of 0.8996 and 0.9246 is obtained between the GPS and IAA, 0.843 and 0.85406 between GPS and ADP, respectively. The high correlation factor acquired is indicating the relative accuracy of the wind speed calculated using IAA method confirming its efficiency and effectiveness. Figures 9 and 10 shows the correlation between IAA and GPS wind speeds.

\section{Conclusion}

An iterative weighted least square-based estimation (IAA) is presented and applied to the atmospheric radar data in the current study. The reduction in the deviation of mean Doppler profiles and the standard deviation is illustrated in IAA compared to the existing ADP. This 

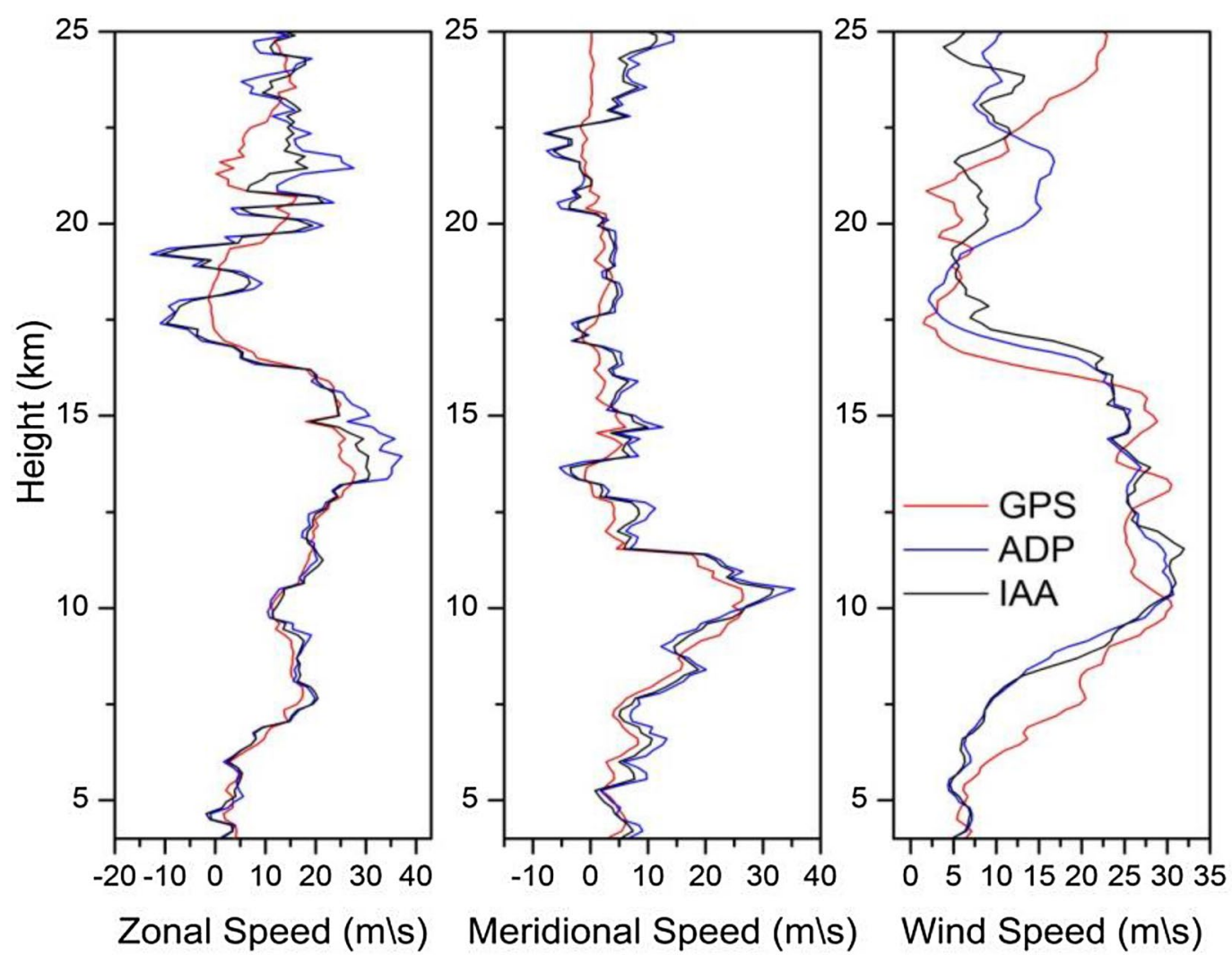

Fig. 7 Zonal, Meridional and Wind Speed for February 9, 2015 data using ADP, IAA and GPS radiosonde

proves that IAA functions superior compared to existing algorithms which have failed to perform well especially in this height range. Significant enhancement in SNR at higher altitudes is achieved with IAA demonstrating its efficiency and effectiveness. The obtained wind velocities from the IAA algorithm are validated using the GPS sonde values. The correlation between wind speeds calculated using GPS and IAA has been also presented for the radar data collected in the month of October 2006 and February 2015 which attained a correlation factor of 0.8996 and 0.9246 . The IAA method gives better results with high computational complexity. This is due to the fact that it is an iterative process involving matrix inversion. Henceforth, this can be carried through efficient 


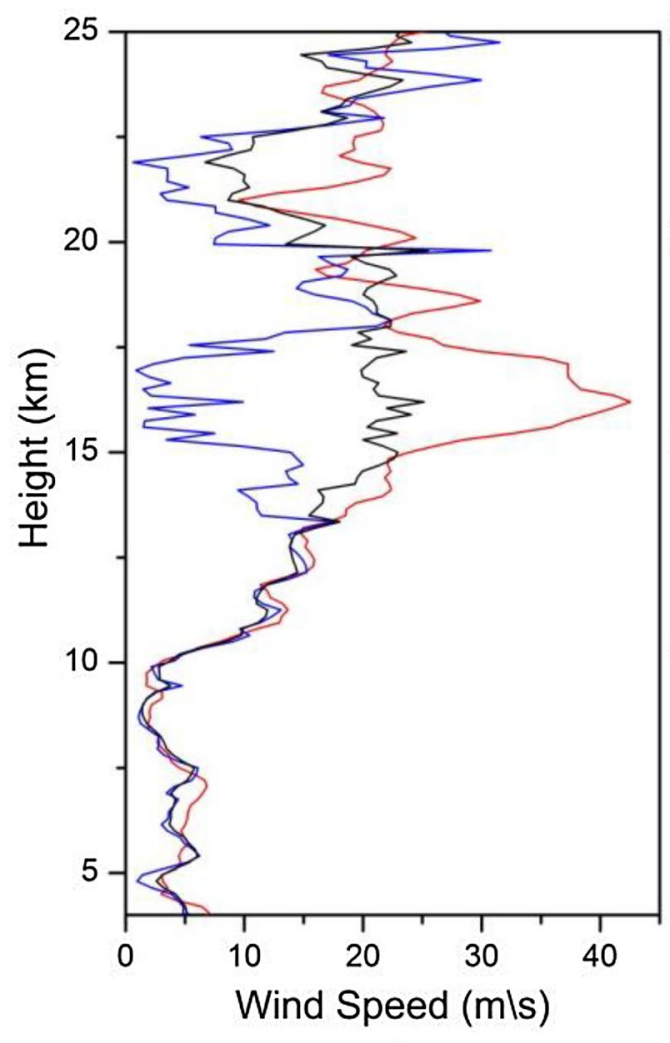

a

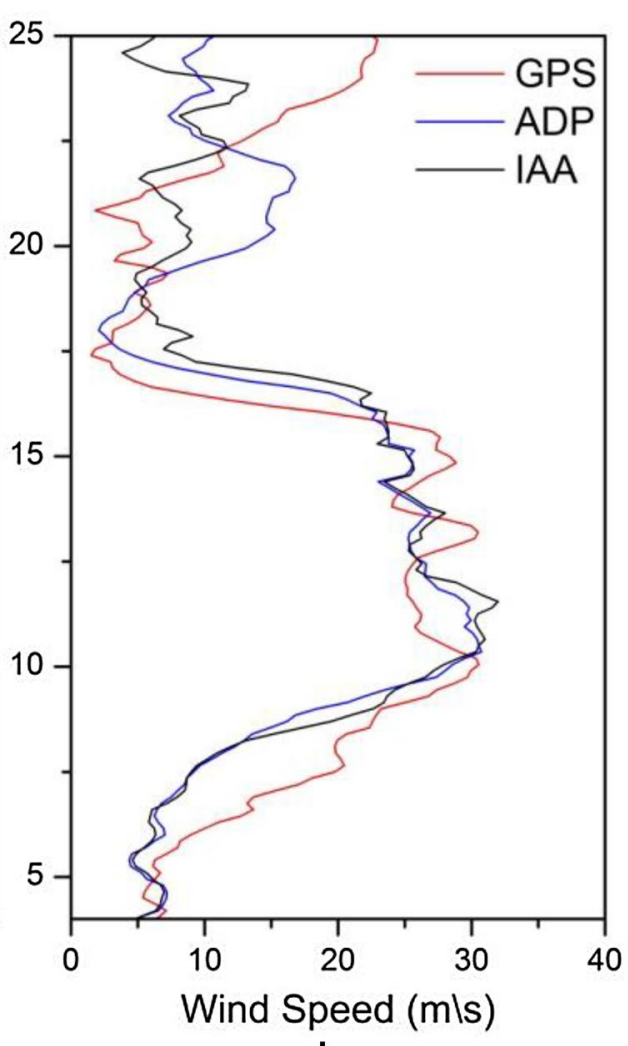

b

Fig. 8 Wind speed using ADP, IAA and GPS radiosonde for radar data collected on a July 2, 2014 b February 9, 2015-Comparison

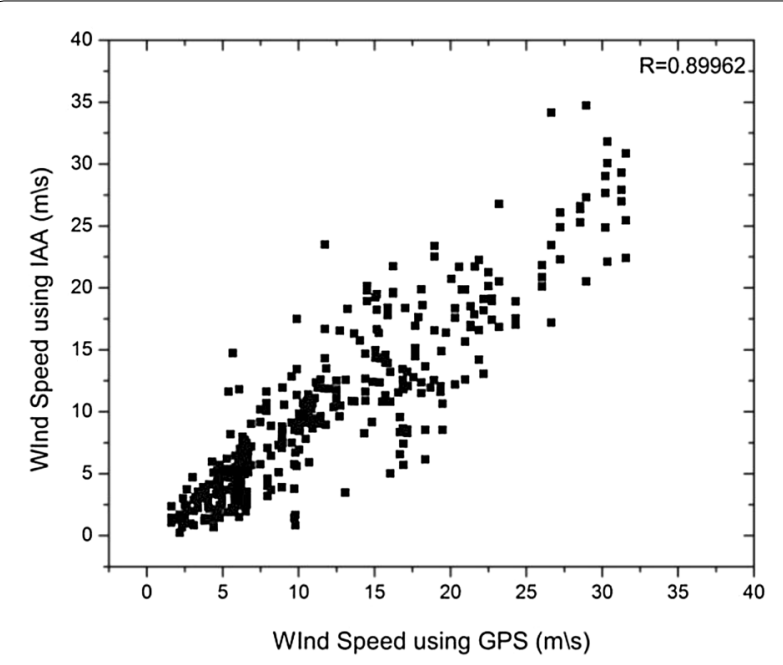

Fig. 9 Correlation between IAA and GPS wind speeds for data during 4th-8th October 2006

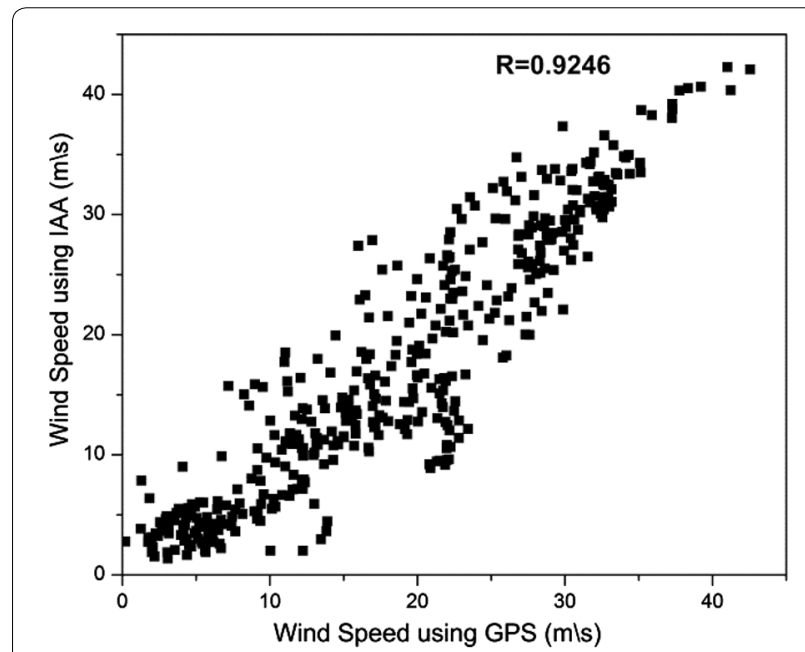

Fig. 10 Correlation between IAA and GPS wind speeds for data during 9th-12th February 2015 
methods which results in consuming less computational time.

\section{Authors' contributions}

Both authors read and approved the final manuscript.

\section{Acknowledgements}

The authors would like to thank National Atmospheric Research Laboratory (NARL), Gadanki for providing radar data and Centre of Excellence, Department of Electronics and Communication Engineering, Sri Venkateswara University College of Engineering for technical assistance.

\section{Competing interests}

The authors declare that they have no competing interests.

\section{Availability of data and materials}

The data is archived at the National Atmospheric Research Laboratory, Gadanki, India.

\section{Funding}

Not applicable.

\section{Publisher's Note}

Springer Nature remains neutral with regard to jurisdictional claims in published maps and institutional affiliations.

Received: 1 June 2018 Accepted: 2 August 2018

Published online: 14 August 2018

\section{References}

Anandan VK (2002) Atmospheric data processor-technical and user reference manual. NMRF, DOS Publication, Gadanki

Anandan VK, Balamuralidhar P, Rao PB, Jain AR (1996) A method for adaptive moments estimation technique applied to MST radar echoes. In: Proceedings of the Progress Electromagnetics Research Symposium, pp 360-365
Anandan VK, Reddy GR, Rao P (2001) Spectral analysis of atmospheric radar signal using higher order spectral estimation technique. IEEE Trans Geosci Remote Sens 39(9):1890-1895

Anandan VK, Pan C, Rajalakshmi T, Reddy GR (2004) Multitaper spectral analysis of atmospheric radar signals. Ann Geophys 22(11):3995-4003

Anandan VK, Balamuralidhar P, Rao P, Jain A (2005) An adaptive moments estimation technique applied to MST radar echoes. J Atmos Ocean Technol 22:396-408

Hildebrand PH, Sekhon R (1974) Objective determination of the noise level in Doppler spectra. J Appl Meteorol 13(7):808-811

Jagannadha Rao VVM, Narayana Rao D, Venkat Ratnam M, Mohan K, Vijaya Bhaskar Rao S (2003) Mean vertical velocities measured by Indian MST radar and comparison with indirectly computed values. J Appl Meteorol 42(4):541-552

Reddy TS, Reddy GR (2010a) MST radar signal processing using cepstral Thresholding. IEEE Trans Geosci Remote Sens 48(6):2704-2710

Reddy TS, Reddy GR (2010b) Spectral analysis of atmospheric radar signal using filter banks polyphase approach. Digit Signal Process 20(4):1061-1071

Thatiparthi S, Gudheti R, Sourirajan V (2009) MST radar signal processing using wavelet-based denoising. IEEE Geosci Remote Sens Lett 6(4):752-756

Uma Maheswara Rao D, Reddy TS, Reddy GR (2014) Atmospheric radar signal processing using principal component analysis. Digit Signal Process 32:79-84

Yardibi T, Li J, Stoica P, Xue M, Baggeroer AB (2010) Source localization and sensing: a nonparametric iterative approach based on weighted least squares. IEEE Trans Aero Electron 46:425-443

\section{Submit your manuscript to a SpringerOpen ${ }^{\circ}$ journal and benefit from:}

- Convenient online submission

- Rigorous peer review

- Open access: articles freely available online

- High visibility within the field

- Retaining the copyright to your article

Submit your next manuscript at $\gg$ springeropen.com 